\title{
Perfect Discrete Morse Functions on Triangulated 3-Manifolds
}

\author{
Rafael Ayala, Desamparados Fernández-Ternero, and José Antonio Vilches \\ Departamento de Geometría y Topología, Universidad de Sevilla, \\ P.O. Box 116041080 Sevilla Spain, \\ \{rdayala, desamfer, vilches\}@us.es
}

\begin{abstract}
This work is focused on characterizing the existence of a perfect discrete Morse function on a triangulated 3-manifold $M$, that is, a discrete Morse function satisfying that the numbers of critical simplices coincide with the corresponding Betti numbers. We reduce this problem to the existence of such kind of function on a spine $L$ of $M$, that is, a 2subcomplex $L$ such that $M-\Delta$ collapses to $L$, where $\Delta$ is a tetrahedron of $M$. Also, considering the decomposition of every 3-manifold into prime factors, we prove that if every prime factor of $M$ admits a perfect discrete Morse function, then $M$ admits such kind of function.
\end{abstract}

Keywords: perfect discrete Morse function, triangulated 3-manifold, spine.

\section{Introduction}

The existence of a perfect discrete Morse function on a triangulated 3-manifold $K$ heavily depends on $\mathrm{K}$ and gives us information about its combinatorial structure. For example, it is easy to prove that if we consider a triangulation of $S^{3}$ containing the Dunce hat as subcomplex then any discrete Morse function defined on it has at least 3 critical simplices. This situation strongly contrasts with the smooth case, where the existence of perfect functions on a manifold does not depend on the given triangulation. The main goal of this work, which is the continuation of the paper [2], consists on reducing the problem, initially stated on 3-manifolds, to 2-complexes. It is possible since we prove that a triangulation $K$ of a 3-manifold admits a perfect discrete Morse function if and only if a spine of $K$ admits such kind of function. For the sake of simplicity, we have restricted our study to closed orientable 3-manifolds.

This problem can be regarded not only in a theoretical way but also it is strongly linked with applications in several areas like digital image processing, object recognition and representation of digital objects. In this sense there are works which use 3-dimensional cell complexes for modelling $4 D$ digital objects, which arise in a natural way when considering a time sequence of $3 D$ objects (see [19] and 5]). Any discrete Morse function provides information on the homology of a manifold. For example, the number of critical $i$-cells is not less than the $i$-th Betti number of the manifold. In this context, a perfect discrete Morse 
function on a complex encodes its minimal topological structure in terms of the basic $n$-cycles. Notice that it is not always possible to get a perfect discrete Morse function, since there are complexes not admitting such kind of functions. For example, there are triangulations of 3-balls containing complicated knots as subcomplexes with few edges not admitting perfect functions (see [4] for details). Optimal discrete Morse functions (those with the minimal number of critical cells) can be used to obtain minimal decompositions of complexes/digital images in terms of homological forests (see [17] for details). Only in the case that the considered complex admits perfect discrete Morse function, such minimal decomposition has as many critical $i$-cells as the $i$-th Betti number and, moreover, the triangulation of the model is "nice", that is, the complex contains no complicated knot (see 4]).

Discrete Morse functions arise in a natural way in the image context as graylevel scale digital images are taken into account. Being more precise, starting from real values (graylevel scale) on the vertices of a cubical complex, they are extended to the complex by defining a discrete Morse function on it (see [21] and [1] $)$.

The paper is organized as follows: Section 2 is devoted to introducing the basic notions and results concerning to discrete Morse theory. Section 3 includes the obtained results on graphs and 2-complexes. Once the main result is obtained at the beginning of Section 4, that is, the reduction of the problem of deciding if a given triangulated 3-manifold admits perfect discrete Morse functions to the 2-dimensional case, we indicate necessary conditions in terms of the homology of the manifold for the existence of perfect functions. Also, taking into account the decomposition of a 3-manifold into prime manifolds, we prove in a constructive way that if every prime factor admits a perfect function then the given manifold admits such a kind of function.

\section{Preliminaries}

Through all this paper we shall consider finite simplicial complexes.

Let $K$ be a simplicial $n$-complex and $\alpha$ be a $n$-simplex of $K$. If there exists a $(n-1)$-dimensional face $\beta$ of $\alpha$ such that $\beta$ is not a face of any other $n$-simplex in $K$, we say that there is an elementary collapse from $K$ to $K-\{\alpha, \beta\}$. The inverse operation is called an elementary expansion from $K-\{\alpha, \beta\}$ to $K$. If $K=K^{0} \supset K^{1} \supset \cdots \supset K^{m}=L$ are simplicial complexes such that there is an elementary collapse from $K^{i-1}$ to $K^{i}, i=1, \ldots, m$, we say that $K$ collapses to $L$, denoted by $K \searrow L$. Equivalently, the inverse operation is called an expansion from $L$ to $K$, denoted by $K \nearrow L$. The notion of collapse was introduced by J.C.H. Whitehead 22 in the context of simple homotopy theory. More recently, there have been introduced weaker notions like shaving in the computation of the homology of cubical complexes [18 and contraction in the computation of cohomology operations [10] which are more suitable for Computational Topology. 
The collapse number of a 2-complex $K$, denoted by $c o(K)$, is the minimal number of 2 -simplices $\tau_{1}, \ldots, \tau_{c o(K)}$ that need to be removed from $K$ so that $K-\left\{\tau_{1}, \ldots, \tau_{c o(K)}\right\}$ collapses to a graph. A detailed study of the computability of this number can be found in [6].

A 2-complex such that every 1-simplex is a face of exactly two 2-simplices is called a 2-pseudomanifold. A 2-complex $K$ is said to be strongly connected if, given any two 2-simplices $\sigma, \sigma^{\prime}$ in $K$, there exists a chain of 2-simplices connecting them, that is, a sequence $\sigma_{0}, \ldots, \sigma_{k}$ of 2 -simplices such that $\sigma=\sigma_{0}$, $\sigma^{\prime}=\sigma_{k}$ and $\sigma_{i} \cap \sigma_{i+1}$ is a common 1-face. The strongly connected components of a 2-complex are its maximal strongly connected subcomplexes. A strongly connected 2-pseudomanifold is called a strong 2-pseudomanifold. Notice that any 2-pseudomanifold $K$ can be decomposed as the union of its strongly connected components and the intersection of two of such components is either empty or a finite set of vertices.

Given a simplicial complex $K$, a discrete Morse function is a function $f: K \longrightarrow \mathbb{R}$ such that, for any $p$-simplex $\sigma \in K$ :

(M1) $\operatorname{card}\left\{\tau^{(p+1)}>\sigma / f(\tau) \leq f(\sigma)\right\} \leq 1$.

(M2) $\operatorname{card}\left\{v^{(p-1)}<\sigma / f(v) \geq f(\sigma)\right\} \leq 1$.

A $p$-simplex $\sigma \in K$ is said to be a critical simplex with respect to $f$ if:

(C1) $\operatorname{card}\left\{\tau^{(p+1)}>\sigma / f(\tau) \leq f(\sigma)\right\}=0$.

(C2) $\operatorname{card}\left\{v^{(p-1)}<\sigma / f(v) \geq f(\sigma)\right\}=0$.

A value of a discrete Morse function on a critical simplex is called critical value.

Given $c \in \mathbb{R}$, the level subcomplex $K(c)$ is the subcomplex of $K$ consisting of all simplices $\tau$ with $f(\tau) \leq c$, as well as all of their faces, that is,

$$
K(c)=\bigcup_{f(\tau) \leq c} \bigcup_{\sigma \leq \tau} \sigma
$$

Given two values of $f, a_{k}<a_{l}$, the relationship between two level subcomplexes $K\left(a_{k}\right)$ and $K\left(a_{l}\right)$ is the following [7]:

If the interval $\left[a_{k}, a_{l}\right]$ does not contain any critical value, then $K\left(a_{l}\right)$ collapses to $K\left(a_{k}\right)$ or equivalently, $K\left(a_{k}\right)$ expands to $K\left(a_{l}\right)$.

If the interval $\left[a_{k}, a_{l}\right]$ contains a critical value corresponding to a critical simplex of dimension $i$, then $K\left(a_{l}\right)$ has the same simple homotopy type as $K\left(a_{k}\right)$ with an $i$-cell attached.

A discrete vector field $V$ on $K$ is a collection of pairs $\left(\alpha^{(p)}<\beta^{(p+1)}\right)$ of simplices of $K$ such that each simplex is in at most one pair of $V$. A $V$-path is a sequence of simplices

$$
\alpha_{0}^{(p)}, \beta_{0}^{(p+1)}, \alpha_{1}^{(p)}, \beta_{1}^{(p+1)}, \ldots, \beta_{r}^{(p+1)}, \alpha_{r+1}^{(p)}, \ldots
$$

such that, for each $i \geq 0$, the pair $\left(\alpha_{i}^{(p)}<\beta_{i}^{(p+1)}\right) \in V$ and $\beta_{i}^{(p+1)}>\alpha_{i+1}^{(p)} \neq \alpha_{i}^{(p)}$.

Given a discrete Morse function $f$ on $K$, the gradient vector field induced by $f$ is the set of pairs of simplices $\left(\alpha^{(p)}<\beta^{(p+1)}\right)$ such that $f(\alpha) \geq f(\beta)$. 
Theorem 1. [8] $A$ discrete vector field $V$ is the gradient vector field of a discrete Morse function if and only if there are no non-trivial closed $V$-paths.

Theorem 2. [7] Let $f$ be a discrete Morse function defined on $K$ and let $b_{p}$ be the $p$-th Betti number of $X$ with $p=0,1, \ldots, n$ (where $n$ is the dimension of $K$ ). Then:

(I1) $m_{p}(f)-m_{p-1}(f)+\cdots+(-1)^{p} m_{0} \geq b_{p}-b_{p-1}+\cdots+(-1)^{p} b_{0}$,

(I2) $m_{p}(f) \geq b_{p}$

(I3) $m_{0}(f)-m_{1}(f)+\cdots+(-1)^{n} m_{n}(f)=b_{0}-b_{1}+\cdots+(-1)^{n} b_{n}=\chi(X)$,

where $m_{p}(f)$ denotes the number of critical p-simplices of $f$ on $K$.

Notice that these inequalities are still valid for the case of Betti numbers with general coefficients, that is, using any field $F$ instead of $\mathbb{Z}$.

In the smooth setting Pitcher proved in [20] the following generalized version of Morse inequalities which also take into account the torsion coefficients $q_{p}$ of $H_{p}(M ; F)$, where $F$ is a field or $\mathbb{Z}$ :

Theorem 3. Let $M$ be a compact Riemannian manifold and let $f$ be a smooth Morse function. Then:

1. $m_{0}(f) \geq b_{0}, m_{p}(f) \geq b_{p}+q_{p}+q_{p-1}$ with $p=1, \ldots, n-1, m_{n}(f) \geq b_{n}+q_{n-1}$.

2. $m_{p}(f)-m_{p-1}(f)+\cdots+(-1)^{p} m_{0} \geq b_{p}-b_{p-1}+\cdots+(-1)^{p} b_{0}+q_{p}$ with $p=1, \ldots, n-1$.

This result can be extended in a straightforward way to the discrete approach.

A discrete Morse function $f$ defined on $K$ is optimal if it has the least possible number of critical simplices, that is, $m_{i}(f) \leq m_{i}(g)$ with $1 \leq i \leq n$ for every discrete Morse function $g$ on $K$.

A discrete Morse function $f$ is called $F$-perfect if $m_{p}(f)=b_{p}(K ; F)$ with $p=0, \ldots, n$. Taking into account the Morse inequality (I2) of Theorem 2, we conclude that every $F$-perfect function is optimal.

Remark 1. Notice that if a discrete Morse function is $\mathbb{Z}$-perfect then, due to $\beta_{i}(K, \mathbb{Z})=\beta_{i}(K, \mathbb{Q})$, the function is $\mathbb{Q}$-perfect too. Moreover, if a complex $K$ admits a $\mathbb{Q}$-perfect discrete Morse function, then the homology groups of $K$ with coefficients in $\mathbb{Z}$ are torsion-free (see Proposition 5.9 of 14 for details). By means of the universal coefficient theorem, the Betti numbers are the same for coefficients in any field and consequently the function is $F$-perfect for all field $F$.

\section{Perfect Discrete Morse Functions on Graphs and 2-Complexes}

We will start the study of the existence of perfect discrete Morse functions on 2-complexes by considering the case of homology with integer coefficients.It is well known that every 1-dimensional complex admits $\mathbb{Z}$-perfect discrete Morse 
functions. It can be proved considering a spanning tree, by means of Lemma 4.3 of [7], a discrete Morse function on such tree with a unique critical vertex is constructed. Finally, this function is extended to the graph by assigning a local maximum to every edge not contained in the tree.

As a direct consequence, taking into account Lemma 4.3 of [7, every 2complex collapsing to a graph admits a $\mathbb{Z}$-perfect discrete Morse function. In particular, since every surface with boundary collapses to a graph (see example 7 , page 52 of [9]), such surfaces admit a $\mathbb{Z}$-perfect discrete Morse function.

Now we are going to give several results on the links between the existence of $\mathbb{Z}$-perfect discrete Morse functions on a given 2-complex with some trivial homology groups and its simple homotopy type.

Proposition 1. Let $K$ be a compact connected 2 -complex admitting a $\mathbb{Z}$-perfect discrete Morse function. The following statements hold:

1. If $K$ is $\mathbb{Z}$-acyclic then it is collapsible.

2. If $H_{1}(K)=0$ and $H_{2}(K) \neq 0$ then $K$ has the same simple homotopy type as a wedge of copies of $S^{2}$.

3. If $H_{1}(K) \neq 0$ and $H_{2}(K)=0$ then $K$ has the same simple homotopy type as a wedge of copies of $S^{1}$.

It follows from the above result that every compact connected 2-complex which is $\mathbb{Z}$-acyclic and non-contractible does not admit $\mathbb{Z}$-perfect discrete Morse functions. An example of such kind of 2-complex can be found in [16].

Using a straightforward Mayer-Vietoris argument, we can prove that every complex $K$ admitting $\mathbb{Z}$-perfect discrete Morse functions satisfies $H_{1}(K ; \mathbb{Z})$ is free. Notice that the converse is not true. In [2] an example of a 2-complex $K$ with $H_{1}(K)=\mathbb{Z}$ and not admitting $\mathbb{Z}$-perfect discrete Morse functions is included. In particular, if the first fundamental group of a 2-complex is finite and non-trivial then it does not admit $\mathbb{Z}$-perfect discrete Morse functions.

By means of the collapse number of a 2-complex we proved that a connected compact 2-complex $K$ admits a $\mathbb{Z}$-perfect discrete Morse function if and only if $c o(K)=b_{2}(K ; \mathbb{Z})$. As a direct consequence we obtained that a compact connected surface without boundary admits a $\mathbb{Z}$-perfect discrete Morse function if and only if it is orientable. Moreover, this result can be extended to 2-pseudomanifolds in the sense that a 2-pseudomanifold $K$ admits a $\mathbb{Z}$-perfect discrete Morse function if and only if every strongly connected component of $K$ is orientable.

\section{Perfect Discrete Morse Functions on 3-Manifolds}

In this section we will consider the problem of the existence of perfect discrete Morse functions on 3-manifolds. First of all, let us recall some basic notions and results concerning 3 -manifolds. 
Definition 1. Given two closed triangulated 3-manifolds $K$ and L, the connected sum of them, denoted by $K \sharp L$, is defined as follows:

- We choose two 3-simplices $\sigma$ and $\tau$ in $K$ and $L$ respectively.

- We identify in $(K-\sigma) \cup(L-\tau)$ the simplices of the boundaries of $\sigma$ and $\tau$ by some simplicial gluing map.

Definition 2. A connected triangulated 3-manifold $M$ is called prime if $M=K \sharp L$ implies $K=S^{3}$ or $L=S^{3}$.

Theorem 4 (Knesser-Milnor[12]). Let $M$ be a compact connected orientable triangulated 3-manifold. Then there is a decomposition $M=P_{1} \sharp \cdots \sharp P_{n}$ with each $P_{i}$ prime. This decomposition is unique up to insertion or deletion of $S^{3}$.

Using the Pitcher's strengthened version of Morse inequalities we get the next result

Proposition 2. Let $M$ be a closed triangulated 3-manifold admitting a $\mathbb{Z}$-perfect discrete Morse function, then $M$ is orientable and $H_{1}(M ; F)$ is free for all field $F$.

Remark 2. The converse of the above result is not true since there are 3-manifolds with $H_{1}(M)$ free which do not admit $\mathbb{Z}$-perfect discrete Morse functions as it is shown in the following example:

Consider the 3-manifold $M=H^{3} \sharp\left(S^{1} \times S^{2}\right)$, where $H^{3}$ denotes the Poincare's homology sphere (see [12 and [13]). Notice that $H_{1}(M)=\mathbb{Z}$ is free (in fact $H_{i}(M)=\mathbb{Z}$ ) and thus a perfect discrete Morse function has 4 critical simplices. However, since the first homotopy group $\Pi_{1}(M)$ is not abelian it follows that any presentation of this group has at least two generators and then the 1-skeleton of any $C W$-structure of $M$ contains the wedge of at least two circles. So $m_{2}=$ $m_{1} \geq 2$ and hence we conclude that every discrete Morse function on $M$ has at least 6 critical simplices.

Corollary 1. Let $M$ be a closed triangulated 3-manifold such that $\Pi_{1}(M)$ is $f$ nite and non-trivial. Then $M$ does not admit $\mathbb{Z}$-perfect discrete Morse functions.

Remark 3. Examples of this kind of spaces are the spherical manifolds $S^{3} / \Gamma$ where $\Gamma$ is a finite subgroup of $S O(4)$ acting freely on $S^{3}$ by rotations. In the particular case that $\Gamma$ is a cyclic group these manifolds are the lens spaces $L(p, q)$ for $0<\frac{p}{q}<1$.

Corollary 2. Let $M$ be a closed triangulated 3 -manifold such that $\Pi_{1}(M)$ contains a torsion subgroup. Then $M$ does not admit $\mathbb{Z}$-perfect discrete Morse functions.

Definition 3. Let $M$ be a triangulated 3-manifold. A spine $L$ of $M$ is a 2subcomplex $L$ such that $M-\Delta$ collapses to $L$, where $\Delta$ is a tetrahedron of $M$. 

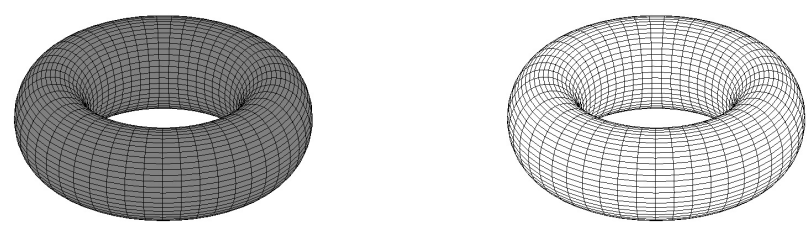

Fig. 1. The figure on the left is solid torus admitting as spine the torus on the right

The next figure represents a spine of the 3-manifold obtained by the complement in a 3-ball of a knotted thickened Y, the so called worm-eaten apple.

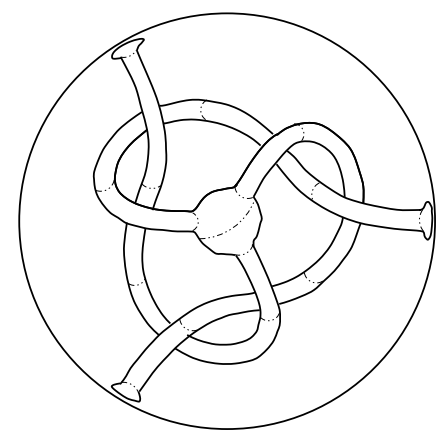

Fig. 2. A spine of a worm-eaten apple

Next result illustrates how the problem of deciding whether a given orientable 3 -manifold $M$ admits a $\mathbb{Z}$-perfect discrete Morse function is reduced to the twodimensional case, that is, it is equivalent to the problem of determining the existence of such kind of functions on a spine of $M$. Algorithms for the obtention of perfect functions on 2-complexes are presented in [3].

Theorem 5. Let $M$ be a connected closed orientable triangulated 3-manifold and let $F$ be either $\mathbb{Z}$ or a field. $M$ admits an $F$-perfect discrete Morse function if and only if there exists $L$, a spine of $M$, which admits an $F$-perfect discrete Morse function.

Proof. Let us suppose that $M$ admits an $F$-perfect discrete Morse function $f$, hence $m_{i}(f)=b_{i}$ with $i=0, \ldots, 3$. Since $H_{3}(M) \simeq \mathbb{Z}$ and $M$ is connected, then $m_{0}(f)=1=m_{3}(f)$. By means of Poincare duality's theorem, we get that $m_{1}(f)=m_{2}(f)$. Let $N$ be the subcomplex obtained by removing the unique critical 3-simplex of $f$ denoted by $\Delta$. Notice that $N$ is of the same simple homotopy type as a 2-dimensional subcomplex $L$, since $N$ can be obtained from $L$ by a sequence of elementary expansions, in fact $L$ is a spine of $M$. The proof can be carried out by induction on the sequence of level subcomplexes taking into account that every 3 -simplex added at a given step provides us an expansion 
(since no 3-simplex is critical). Then, by an easy Mayer-Vietoris argument we get $b_{i}(L)=b_{i}(M-\Delta)=b_{i}(M)$ with $i=0,1,2$. Indeed the restriction $g$ of $f$ to $L$ is perfect satisfying $m_{i}(f)=m_{i}(g)$ with $i=0,1,2$.

Conversely, let us assume that there is a spine $L$ admitting an $F$-perfect discrete Morse function $g$. Let $\Delta$ be a 3-simplex such that $M-\Delta \searrow L$ then by a Forman's result (Lemma 4.3 of [7]), $g$ can be extended to a $\mathbb{Z}$-perfect discrete Morse function $\widehat{g}$ on $M-\Delta$. Finally, we extend $\widehat{g}$ to $M$ by defining $f(\Delta)=1+\max _{\sigma<\Delta} f(\sigma)$.

The following result guarantees the existence of a perfect discrete Morse function on a closed 3-manifold if every single component of its prime factor (given by Theorem 4) admits such a kind of function.

Theorem 6. Let $M=M_{1} \sharp M_{2}$ be a decomposition of a connected closed orientable 3-manifold $M$ and let $F$ be either $\mathbb{Z}$ or a field. If there exist triangulations $K_{i}$ of $M_{i}$ admitting an $F$-perfect discrete Morse function with $i=1,2$ then there exists a triangulation $K$ of $M$ admitting an $F$-perfect discrete Morse function.

Proof. Let us assume that $K_{i}$ is a triangulation of $M_{i}$ admitting an $F$-perfect discrete Morse function with $i=1,2$. By using Theorem 5 , there are spines $L_{i}$ of $K_{i}$ admitting $F$-perfect discrete Morse functions $f$ and $g$. Let $L_{1} \vee L_{2}$ be the wedge obtained by identifying the only critical vertices $v$ and $w$ of $f$ and $g$ respectively. Then the function $h$, defined as

$$
h(\sigma)= \begin{cases}f(\sigma), & \text { if } \sigma \in L_{1}-\{v\} \\ g(\sigma), & \text { if } \sigma \in L_{2}-\{w\} \\ \min \{f(v), g(w)\}, & \text { if } \sigma=v=w\end{cases}
$$

is an $F$-perfect discrete Morse function on $L_{1} \vee L_{2}$.

Since it is known that $L_{1} \vee L_{2}$ is a spine of $K=K_{1} \sharp K_{2}$, (see [15]) then by means Theorem 5, we conclude that $K$ (which is a triangulation of $M$ ) admits an $F$-perfect discrete Morse function.

Remark 4. Taking into account the classification of closed prime 3-manifolds based on their fundamental group (see [13]), by Corollary 1 we obtain that a closed prime 3-manifold $M$ admitting a perfect discrete Morse function is either $S^{1} \times S^{2}$ or $K\left(\Pi_{1}(M), 1\right)$ where $\Pi_{1}(M)$ is infinite but not cyclic.

In this second case, by means of Corollary 2 we conclude that $\Pi_{1}(M)$ is torsion free and also, if we suppose that $\Pi_{1}(M)$ is abelian then $M=S^{1} \times S^{1} \times S^{1}$. Now, since $H_{1}(M)$ is infinite then $M$ must be a sufficiently large Haken manifold, for example, $M_{g} \times S^{1}$ where $M_{g}$ is an orientable surface of genus $g$ (see [13]).

\section{References}

1. Ayala, R., Fernández, L.M., Vilches, J.A.: Discrete Morse inequalities on infinite graphs. Electron. J. Comb. 16 (1), paper R38, 11 (2009)

2. Ayala, R., Fernández-Ternero, D., Vilches, J.A.: Perfect discrete Morse functions on 2- complexes. Pattern Recognition Lett. (2011), doi: 10.1016/j.patrec.2011.08.011 
3. Ayala, R., Fernández-Ternero, D., Vilches, J.A.: Constructing optimal discrete Morse functions on certain 2-complexes (submitted)

4. Benedetti, B.: Discrete Morse Theory is as perfect as Morse Theory. arXiv:1010.0548v3 [math.DG]

5. Couprie, M., Bertrand, G.: New characterizations of simple points in 2D, 3D and 4D discrete spaces. IEEE Trans. Pattern Anal. Mach. Intell. 31(4), 637-648 (2009)

6. Ĕgecioğlu, Ö., Gonzalez, T.: A computationally intractable problem on simplicial complexes. Comput. Geom. 6(2), 85-98 (1996)

7. Forman, R.: Morse Theory for cell complexes. Adv. Math. 134(1), 90-145 (1998)

8. Forman, R.: A user's guide to discrete Morse theory. Sém. Lothar. Combin. 48, Art. B48c, 35 (2002) (electronic)

9. Glaser, L.C.: Geometrical Combinatorial Topology. Van Nostrand Reinhold Company, New York (1970)

10. González-Díaz, R., Real, P.: Computation of cohomology operations on finite simplicial complexes. Homology Homotopy Appl. 5(2), 83-93 (2003)

11. Gyulassy, A., Bremer, P.T., Hamann, B., Pascucci, V.: A practical approach to Morse-Smale complex computation: Scalability and generality. IEEE Trans. Vis. Comput. Graph. 14(6), 1619-1626 (2008)

12. Hatcher, A.: Notes on basic 3-manifold Topology, http://www . math. cornell. edu/ hatcher/3M/3Mdownloads.html

13. Hatcher, A.: The clasification of 3-manifolds. A brief overview, http://www . math. cornell.edu/ hatcher/Papers/3Msurvey.pdf

14. Jonsson, J.: Simplicial complexes of graphs. Lecture Notes in Math., vol. 1928. Springer, Berlin (2008)

15. Knutson, G.W.: A characterization of closed 3-manifolds with spines containing no wild arcs. Proc. Amer. Math. Soc. 21, 310-314 (1969)

16. Lutz, F., Ziegler, G.: A small Polyhedral $\mathbb{Z}$-Acyclic 2-complex in $\mathbb{R}^{4}$. EG-Models, No. 2008.11.001 (2008)

17. Molina-Abril, H., Real, P.: Homological Spanning Forest Framework for 2D Image Analysis. To Appear in Ann. Math. Artif. Intell.

18. Mrozek, M., Pilarczyk, P., Żelazna, N.: Homology algorithm based on a cyclic subspace. Comput. Math. Appl. 55, 2395-2412 (2008)

19. Pacheco, A., Mari, J.L., Real, P.: Obtaining cell complexes associated to four dimensional digital objects. Imagen-A 1, 57-64 (2010)

20. Pitcher, E.: Inequalities of critical point theory. Bull. Amer. Math. Soc. 64, 1-30 (1958)

21. Robins, V., Wood, P.J., Sheppard, A.P.: Theory and algorithms for constructing discrete Morse complexes from grayscale digital images. IEEE Trans. Pattern Anal. Mach. Intell. 33(8), 1646-1658 (2011)

22. Whitehead, J.H.C.: Simple homotopy types. Amer. J. Math. 72, 1-57 (1950) 